\title{
ANALYSIS OF CRACKS DEVELOPMENT IN ROCK MASSIF WITH THE USE OF DYNAMIC DESTRUCTION RESISTANCE
}

\author{
Anatolii Kriuchkov', Anatolii Bakhtyn ${ }^{2}$ \\ ${ }^{1}$ Department of Geo-Engineering, National Technical University of Ukraine «Igor Sikorsky Kyiv Polytechnic Institute», Kyiv, Ukraine \\ t1104i@ukr.net \\ ORCID: https://orcid.org/0000-0002-2234-0546 \\ ${ }^{2}$ Department of Ecology and Plant Polymers Technology, National Technical University of Ukraine «Igor Sikorsky Kyiv Polytechnic \\ Institute», Kyiv, Ukraine \\ atbahtin.anatolii1994@gmail.com \\ ORCID: https://orcid.org/0000-0002-7081-2894
}

\begin{tabular}{l} 
A R T I L E INF O \\
\hline Article history: \\
Received date 03.03 .2021 \\
Accepted date 15.04 .2021 \\
Published date 30.04 .2021 \\
Section: \\
Geotechnical Engineering \\
D O I \\
10.21303/2313-8416.2021.001695
\end{tabular}

$10.21303 / 2313-8416.2021 .001695$

KEYWORDS

crack

principle of least action

Lagrange equations

dynamic resistance

deformation

crack dynamics

\section{ABSTRACT}

The object of research: the process of development of main cracks in the massif of rocks under the action of a wave impulse taking into account the dynamic destruction resistance. Investigated problem: Description of the dynamics of crack development in the impulse mode of increasing their size, as well as the transition to an unstable mode of crack growth. The main scientific results: In the present article uses an approach based on the principle of least action and $\mathrm{N}$-characteristic of dynamic crack growth resistance. This allowed to obtain analytical equations of the crack growth trajectory in the rock massif and the main characteristics of the process.

The area of practical use of the research results: The considered approach allows to predict the formation of the shear surface in quarries and natural slopes. This approach also allows to set a time-varying probability of their steady state and adjust, if necessary, the parameters of blasting.

Innovative technological product: Analytical regularities for the driving force of the crack and dynamic destruction resistance are obtained, which take into account the velocity and acceleration of the crack, as well as the time and nonstationarity of the impulse load.

Scope of the innovative technological product: The proposed approach can be used in the design of blasting operations in quarries and in the calculation of the probabilities of the steady state of natural massifs under the influence of impulse loads.

(C) The Author(s) 2021. This is an open access article under the Creative Commons CC BY license

\section{Introduction}

During mass explosions in quarries and mining enterprises, the lithosphere is subjected to geomechanical action, which leads to changes in physical and mechanical and morphological properties of the terrain, cracks and cavities, landslides, changes in water regime of the terrain, damage to infrastructure.

According to numerous scientific studies, it is known that the main destructive factor of the lithosphere near mining enterprises is an explosive wave, which in the rocks massif turns into a wave of deformation $[1,2]$.

Cracks in lithosphere objects are violations of the monolithic nature of the rock mass that separates the rock zones with broken interatomic bonds and the area of intact rock.

In the formation and change of rocks in the general process of development of the earth's crust rocks are divided by cracks into blocks of different sizes and shapes. This phenomenon is called fractures of the rock mass. Fracture is a common property of the rock mass. This parameter characterizes the morphological structure, geomechanical properties of the massif and mining and geological conditions of the studied areas of the earth's crust.

Since the fracture of the rock massif is a constant factor influencing the conditions of mining, it must be systematically studied, documented and accounted for.

\section{1. The object of research}

The object of research is the process of development of main cracks in the massif of rocks under the action of a wave impulse, taking into account the dynamic destruction resistance. 


\section{2. The problem}

A charge explosion simulates a small earthquake, the hypocenter of which is located in the place location of the charge. Under the action of an explosive impulse in the rock there is a shock wave, the intensity of which decreases sharply with increasing distance from the center of the explosive charge. At a short distance from the charge are formed zones of plastic and elastic deformations.

Deformation of the rock under the action of the explosion leads to the formation, development, growth and connection of cracks. As of today, the most effective analytical tool for studying the behavior of crack growth is the methods of linear fracture mechanics. The use of these methods in studying the growth dynamics of cracks is based on the concept of quasi-elastic destruction. In this case, it is assumed that under the action of the field of external load, the plastic core of the crack tip moves together with the crack $[3,4]$. However, it should be noted that the destruction of the rock massif occurs in this core under the action of elastic deformation of the object as a whole. It is logical to assume that at significant impulse loads the crack velocity will increase, so the effect of plastic deformations will decrease, but it is necessary to take into account the dynamics of cracks.

In almost all cases to describe the crack motion is used in various modifications the energy balance method Consider a closed surface BS that delimits an arbitrarily deformable region of body $\mathrm{B}$ with a crack, the area of which is CS, and a plastic core in front of a crack C (Fig. 1). According to the law of conservation of energy $[5,6]$ :

$$
W+Q_{\infty}=U_{\infty}+U_{T}+P_{T}+T_{T}+E_{T},
$$

where $W$ - work of external forces; $Q_{\infty}$ - thermal energy that is supplied to the body; $E_{T}-$ destruction energy, which goes to increase the crack surface; $U_{T}$ - thermal energy released in the zone of deformation and destruction; $U_{\infty}$ - energy of elastic deformations of the body and the crack core; $P_{T}$ - energy of plastic deformations of the body and the crack core; $T_{T}$ - kinetic energy of body particles in the area of the crack core.

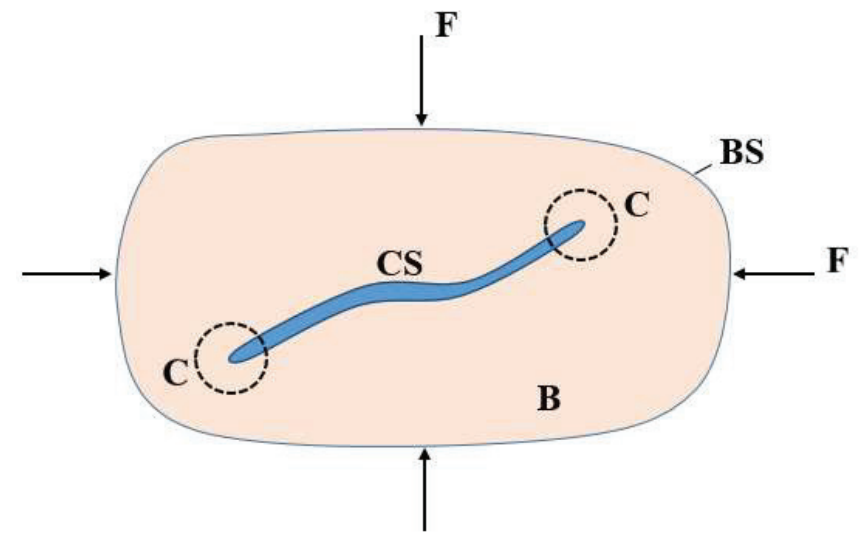

Fig. 1. Conditional body with a crack

Assuming that the array that is subject to destruction is adiabatically isolated and has the same temperature everywhere, so there is no heat flow in the body. Dividing the change in energy by the increase per unit area of the crack surface during its growth, let's obtain a known ratio $G(S)=R(S)[7]$, where

$$
\begin{aligned}
& G(S)=\frac{\partial W}{\partial S}-\frac{\partial U_{\infty}}{\partial S}-\frac{\partial U_{T}}{\partial S}-\frac{\partial T_{T}}{\partial S}, \\
& R(S)=\frac{\partial P_{T}}{\partial S}+\frac{\partial E_{T}}{\partial S},
\end{aligned}
$$

$G(S)$ - dynamic energy release rate, or its equivalent driving force of the crack energy release rate, or its equivalent driving force of the crack; $R(S)$ - crack growth resistance. 
This approach is based on the fact that with the stable development of cracks there is a continuous balance between the force $G(S)$ and the resistance $R(S)$ of course, the concept of R-characteristics $[8,9]$ has a number of advantages, but it is not without drawbacks. The main disadvantages are that this concept applies only to rectilinear cracks and does not take into account the time-varying impulse load and the effect of crack speed on $R(S)$.

\section{3. Analysis of recent research and publications}

At the moment, several solutions of crack dynamics problems are proposed by different methods and with different accuracy. The mechanics of destruction originate from the works [10]. J. Irwin proposed a method of testing and calculation to assess the bearing capacity of a sample containing a crack of known length [11]. Continuations of scientific research proposed by J. Irwin are presented in $[12,13]$.

Mott [14] first determined the crack velocity in a fragile body. In [15], Joffe obtained an important result about the existence of a limit crack velocity approximately equal to $0.3 C_{s}\left(C_{s}\right.$ is the velocity of transverse seismic waves). When this velocity is reached, the trajectory of the cracks begins to warp. The influence of velocity in the dynamics of crack growth was studied in [16, 17]

\section{4. Proposed solution to the problem}

Impulse influence on the system leads to periodic loading and formation of so-called fatigue cracks. The growth of fatigue cracks is intermittent and gradual and with each impulse, their length increases by a certain amount, after the cessation of loading their growth stops. This is a non-stationary process, so the study of the nature of the development of cracks should be based on their dynamics.

The aim of research: Obtaining and analysis of dynamic, nonstationary, nonlinear model of crack development in solid systems using the principle of least action and N-characteristics of dynamic resistance.

Research objectives: of cracks;

1) formulate and describe the principle of least action in assessing the dynamic development

2) develop a mathematical model of the driving force of crack growth, taking into account its velocity and acceleration;

3) obtain, describe and investigate the equation of dynamic crack growth resistance using the N-characteristic.

Our proposed approach is that the general scientific principle of least action is used for the analytical description of the dynamics of crack development in an array of rocks at variable loads.

\section{Materials and Methods}

The values of the dynamic resistance to crack growth in solid objects with bifraction are based on experimental measurements of the change in the crack velocity depending on the intensity of the applied loads $[18,19]$. Isochrome photoelastic paintings were recorded using a high-speed spark chamber. These paintings were obtained by the method of photoelasticity at the time of crack propagation in samples with the same lateral notch under central loading, eccentric application of load and under loading along the crack line. Experimental data obtained for each solid material in several studies cover the growth of cracks at speeds from the limit to that corresponding to the stopping moment.

\section{1. The principle of least action in assessing the dynamic development of the crack}

The principle of least action formulated by Lagrange and improved by Hamilton and Ostrogradsky is that of all possible ways of system development in the configuration space, the one that corresponds to the minimum value of action is realized.

To describe the propagation of body points together with the crack under the influence of impulse loads, it is possible to use classical methods of analytical mechanics. This is realized by introducing into the equation of propagation parameters that take into account the action of external and internal forces, provided that the processes of internal dissipation are much slower than the rate of deformation of the system. 
In our problem, the principle of the least action sounds as follows: from all possible trajectories of development of a crack in a firm body, in practice only that trajectory which is an extremal of a functional is realized:

$$
D=\int_{t_{1}}^{t_{2}}[L(q, \dot{q}, t)-\Phi(q, t)] \mathrm{d} t,
$$

where $L(q, \dot{q}, t)$ - Lagrange function of the second kind for cracks; $\Phi(q, t)$ - generalized non-potential force function, which takes into account the external action and energy dissipation; $q$ - generalized coordinate of the trajectory of the crack vertex; $\dot{q}$ - generalized coordinate of the crack velocity.

From the principle of least action for our problem follows the Lagrange equation of the second kind [20]:

$$
\frac{d}{d t}\left(\frac{\partial L}{\partial \dot{q}}\right)-\frac{\partial L}{\partial \dot{q}}=\sum Q_{i j}, \quad i=1,2,3,
$$

where $Q_{i j}$ - generalized non-potential force.

\section{Results and experimental confirmation}

Thus, according to the principle of least action, the motion of a crack in a solid can be described by the Lagrange equation of the second kind, if the Lagrange function $L(q, \dot{q}, t)$ for cracks and the generalized nonpotential force $Q_{i j}$ acting on it are known. Given the invariance of the Lagrange equation, let's obtain a system of equations that describe the crack motion for a plane problem in the Cartesian coordinate system:

$$
\left\{\begin{array}{l}
\frac{\partial}{\partial a_{x}}\left(U_{\sigma-a}+T_{\sigma-a}\right)-\frac{d}{d t}\left(\frac{\partial T_{\sigma-a}}{\partial \dot{a}_{x}}\right)=N_{x}\left(\dot{a}_{x}\right) \\
\frac{\partial}{\partial a_{y}}\left(U_{\sigma-a}+T_{\sigma-a}\right)-\frac{d}{d t}\left(\frac{\partial T_{\sigma-a}}{\partial \dot{a}_{y}}\right)=N_{y}\left(\dot{a}_{y}\right),
\end{array}\right.
$$

where $U_{\sigma-a}, T_{\sigma-a}$ - potential and kinetic energy of the crack, respectively; $N_{x}\left(\dot{a}_{x}\right) N_{y}\left(\dot{a}_{y}\right)$ - projection of the crack resistance vector on the $X$ and $Y$ axes, respectively; $a_{x}, a_{y}$-projections of the half-length of the crack on the $X$ and $Y$ axes, respectively; $\dot{a}_{x}, \dot{a}_{y}$-projections of the crack velocity on the $X$ and $Y$ axes, respectively.

\section{1. The driving force of crack growth}

For the development of a crack, it is necessary that the system has elements that shift the center of the core in front of the crack. In our problem, an external impulse load performs this function, which is the main factor in the driving force of crack growth.

Mathematically, the driving force of a crack is the ratio of energy loss, at a certain point, to the area of the crack [11]. From a physical point of view, this value is the flow of energy that is required to destroy the material at the top of the crack:

$$
J_{i}=\frac{\partial}{\partial a_{i}}\left(U_{\sigma-a}+T_{\sigma-a}\right)-\frac{d}{d t}\left(\frac{\partial T_{\sigma-a}}{\partial \dot{a}_{i}}\right),
$$

but in our case it depends on the crack velocity, which is included in equation (5) due to the kinetic energy.

The obtained equations of crack motion contain a number of significant differences from the existing ones. These equations allow to describe the motion of not only rectilinear cracks, but also curvilinear ones. The right part of equations (4) is the dynamic resistance of the material to crack propagation, which depends on its velocity. Equation (4) shows that to determine the driving force of the crack $J_{i}$ it is necessary and sufficient to know the kinetic $T_{\sigma-a}$ and potential $U_{\sigma-a}$ energy at any time. 
For our problem at pulsed external loading the driving force of a crack is defined by the equation which considers its dynamics:

$$
J_{x}=\frac{\pi\left(1-v^{2}\right)}{E} \sigma_{1}^{2} a_{x}\left[1-2\left(\frac{\dot{a}_{x}}{V_{m}}\right)\left(1+\frac{\ddot{a}_{x} / \dot{a}_{x}}{\dot{a}_{x} / a_{x}}\right)\right],
$$

where $\sigma_{1}$ - the main maximum tensile stress in the area of the crack tip; $a_{x}$ - projection of the halflength of the crack on the $X$ axis; $\dot{a}_{x}$ - projection of the crack velocity on the $X$ axis; $\ddot{a}_{x}$ - projection of crack acceleration on the $X$ axis; $v$ - Poisson's ratio; $E$ - elastic modulus; $V_{m}$ - the maximum crack velocity in a particular rock.

For a static crack of the first kind $\left(\ddot{a}_{x} \rightarrow 0\right)$, equation (6) has the classical form [11]:

$$
J_{x}=\frac{\pi\left(1-v^{2}\right)}{E} \sigma_{1}^{2} a_{x}
$$

Analysis of the obtained equations allows to draw the following conclusions:

At the time of the appearance of the radial components of the driving force, the trajectory of the crack $J_{i}$ will be significantly distorted. At this point, the system becomes unstable and the movement of the crack in a straight trajectory becomes impossible. The appearance of even slight deviations from the straight line will lead to further distortions of its trajectory.

The dependence of the driving force on its velocity is quite complex. With a constant increase in the external impulse load, the driving force, for a rectilinear crack, decreases linearly with increasing velocity (Fig. 2).

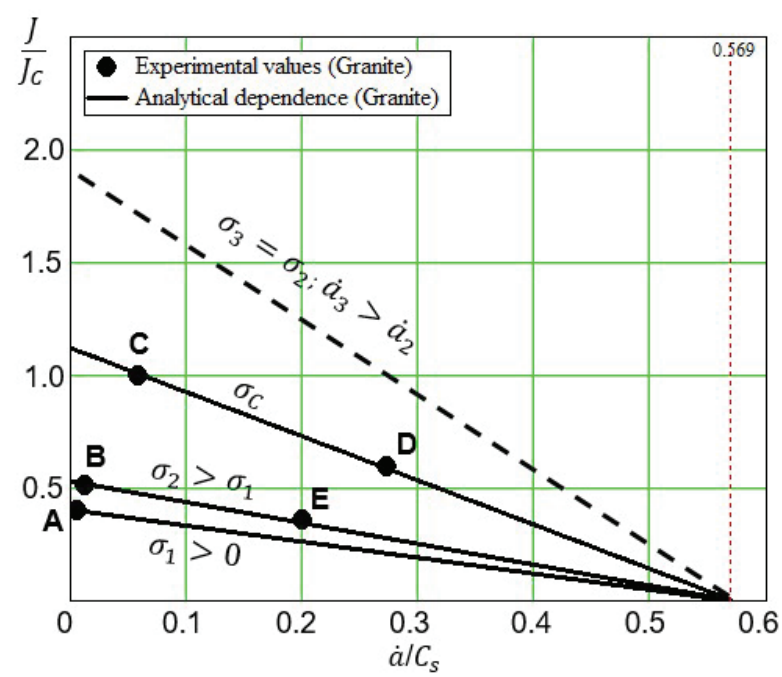

Fig. 2. The driving force of crack development

\section{2. Dynamic crack growth resistance}

Any motion of mass in space receives resistance from the medium and this resistance must be taken into account.

Under the resistance of the crack growth material we understand the pseudovector $\vec{N}$, which is due to the irreversible conversion of the energy of the elastic deformations field into the energy of plastic deformations in the immediate vicinity of the crack apex, as well as kinetic, potential and dissipative energy in the formation of new crack surfaces.

Analytical dependence of the dynamic resistance of crack growth on its velocity for various materials, including rocks, has not yet been obtained, so experimental observations have been used.

The most probable dependence of the crack growth resistance on its velocity is the N-characteristic. An example of such characteristics is calculated from experimental data [18] and is shown in Fig. 3. 
The dependence analysis indicates that this N-characteristic of the dynamic crack growth resistance, under pulse loading, can be described by a fifth degree polynomial:

$$
\frac{N(\dot{a})}{N_{C}}=A_{0}+A_{1}\left(\frac{\dot{a}}{C_{S}}\right)+A_{2}\left(\frac{\dot{a}}{C_{S}}\right)^{2}+A_{3}\left(\frac{\dot{a}}{C_{S}}\right)^{3}+A_{4}\left(\frac{\dot{a}}{C_{S}}\right)^{4}+A_{5}\left(\frac{\dot{a}}{C_{S}}\right)^{5},
$$

where $A_{0}, A_{1}, A_{2}, A_{3}, A_{4}, A_{5}$ - empirical coefficients (Table 1); $\dot{a}$ - crack velocity, $\mathrm{m} / \mathrm{s} ; C_{S}$ - velocity of transverse seismic waves, $\mathrm{m} / \mathrm{s} ; N_{C}$ - critical value of resistance at which unstable crack growth begins, $\mathrm{N} / \mathrm{m} ; J_{C}$-value of the driving force that leads to unstable crack growth, $\mathrm{N} / \mathrm{m}$.

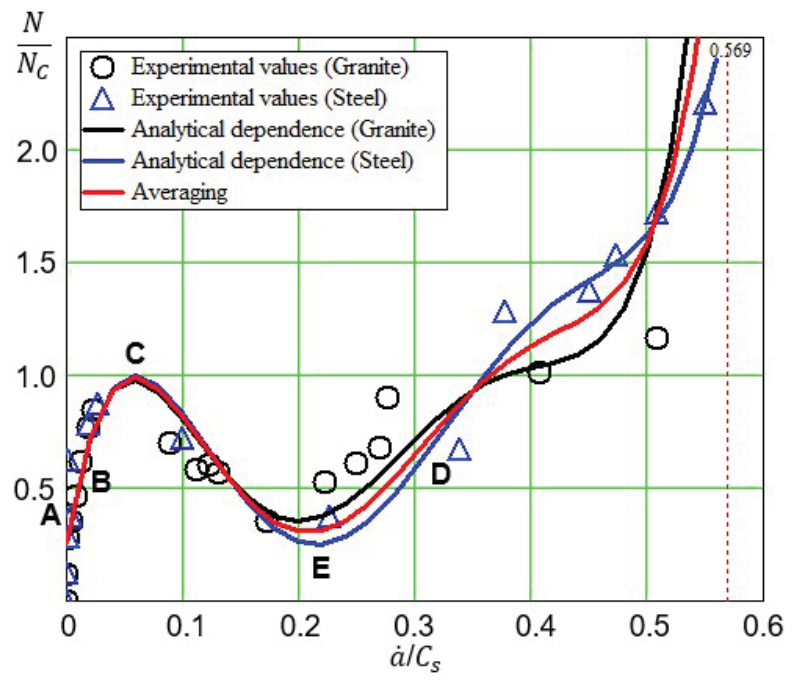

Fig. 3. N-characteristic of the dynamic resistance to crack growth

Table 1

Coefficient of crack growth dependence $N(\dot{a})$

\begin{tabular}{cccccccc}
\hline \multirow{2}{*}{ Material } & \multicolumn{9}{c}{ The value of the coefficients } \\
\cline { 2 - 9 } & $\boldsymbol{A}_{\mathbf{0}}$ & $\boldsymbol{A}_{1}$ & $\boldsymbol{A}_{\mathbf{2}}$ & $\boldsymbol{A}_{\mathbf{3}}$ & $\boldsymbol{A}_{4}$ & $\boldsymbol{A}_{\mathbf{5}}$ & $\boldsymbol{R}^{\mathbf{2}}$ \\
\hline Steel & 0.25 & 30.01 & -387.6 & 1753.4 & -3261.8 & 2174.3 & 0.95 \\
Granite & 0.25 & 30.5 & -415.4 & 2040.9 & -4186.1 & 3085.7 & 0.73 \\
Averaging & 0.25 & 30.26 & -401.5 & 1897.2 & -3724.0 & 2630.0 & 0.85
\end{tabular}

\section{Discussion}

The N-characteristic of crack growth resistance as a consequence of using the principle of least action in the dynamics of crack development was considered by the authors for the first time.

The advantage of the proposed approach is that it takes into account the nonstationarity and dynamics of the crack, in contrast to the R-characteristics [8, 9]. The proposed approach expands the knowledge of the mechanics of the formation of fatigue cracks in a solid body. The concept of $\mathrm{R}$-characteristics $[8,9]$ is fulfilled only to rectilinear, static cracks, while the N-characteristic is adequate for both rectilinear and curvilinear cracks.

Let's analyse the process of development and inhibition of crack growth (Fig. 3). At point 0 , the intensity of the system stress is due solely to internal forces, with increasing external load, the crack velocity slowly increases to point $A$. At point $B$, with increasing load (driving force) stops the growth of the zone of plastic deformation and begins a slow steady growth of cracks. Part of the $O B$ schedule corresponds to the incubation period. Part of the $B C$ graph corresponds to a period of steady crack growth at low velocity. At point $C$, the balance of driving force and dynamic resistance is established $(J=N)$. With further increased load, the crack begins to develop with acceleration and instantly moves to point $D$. If at point $D$ the load stops, the crack velocity instantly returns to point $C$. If the load increases, the crack growth will continue with increasing velocity.

At point $E$, the crack growth is inhibited, the driving force and resistance are balanced, if the load on the system stops, it returns to the starting point $B$, thus completing the full cycle and the 
crack stops. After the first cycle, the crack length increases compared to the previous state. At the subsequent pulse loading growth of a crack is repeated again. As a result, fatigue cracks are formed in the rock massif. In quarries and soil massifs, with continued impulse loading, the crack lengths will increase, forming a shear surface. When they reach a certain critical value, these cracks will begin to coalesce, which can lead to landslide and destruction of the protected object.

The limitations of the research are that the N-characteristics of different materials are obtained as a result of experiments and do not have a single analytical description, which is based on a theoretical basis. At present, the authors see the continuation of research in the development of a physical and mathematical model that would describe the dynamics of crack development based on theoretical ideas about the destruction of solids.

\section{Conclusions}

It is shown that the approach using the principle of least action allows to consider the dynamics of crack development in the array in the form of the Lagrange equation of the second kind.

The obtained equation allows to find the analytical expression $J(\sigma, a, \dot{a}, \ddot{a}, t)$ for the driving force of the crack and to analyze the influence of both the dynamics of the external load $\sigma(\mathrm{t})$ and the dynamics of the crack itself (Fig. 2).

The resistance of the massif is presented by the results of experimental studies (Fig. 3) in the form of an algebraic polynomial on the crack velocity, which allows to take into account the dynamics of crack development under the influence of a time-varying impulse.

The solution of the problem was obtained by analytical-graphical method (Fig. 3), which allowed to analyse the mode of crack development during the passage of a seismic wave in rock massif.

The qualitative and quantitative analysis, as well as the comparison of analytical and experimental data on the development of cracks confirms the possibility of the proposed approach to the study of crack dynamics in a solid. The proposed method is based on the general scientific principle of least action and the method of $\mathrm{N}$-characteristics of the dynamic resistance of the crack.

\section{References}

[1] Frolov, O. O., Kosenko, T. V., Vashchuk, V. Z. (2016). Study of the distribution of energy flows of explosionsin the process of destruction of the model medium. Visnyk NTUU «KPI». Seriia «Hirnytstvo», 30, 23-29. Available at: http://mining.kpi.ua/ article/view/68472/0

[2] Kriuchkov, A., Bakhtyn, A. (2019). Description of the form, dissemination and damping of the explosive impulse in the rocks massif. Up-to-Date Resource- and Energy- Saving Technologies in Mining Industry, 1 (23), 18-27. doi: http://doi.org/ 10.30929/2074-1537.2019.1.18-27

[3] Panin, V. E., Egorushkin, V. E. (2015). Basic Physical Mesomechanics of Plastic Deformation and Fracture of Solids as Hierarchically Organized Nonlinear Systems. Physical Mesomechanics, 18 (4), 377-390. doi: http://doi.org/10.1134/s1029959915040104

[4] Zhang, S., Liu, W., Lv, H. (2019). Creep energy damage model of rock graded loading. Results in Physics, 12, 1119-1125. doi: http://doi.org/10.1016/j.rinp.2018.12.081

[5] Tishchenko, S. V., Eremenko, G. I., Malykh, D. Yu. (2014). Efficiency of explosion energy use when blasting a well charge with explosives. Mining Bulletin, 97, 19-21. Available at: http://nbuv.gov.ua/UJRN/girvi_2014_97_7

[6] Isaiev, S. D., Pashkov, A. P., Fedorenko, P. Y., Napadovska, L. A. (2012). Doslidzhennia efektyvnosti zastosuvannia donnykh prostrilochnykh zariadiv na karierakh. Visnyk NTUU «KPI». Seriia «Hirnytstvo», 22, 109-114. Available at: http://ekmair. ukma.edu.ua/bitstream/handle/123456789/2669/Pashkov_Dosldizhennia_efektyvnosti_zastosuvannia.pdf?sequence=1

[7] Pippan, R., Hohenwarter, A. (2017). Fatigue crack closure: a review of the physical phenomena. Fatigue \& Fracture of Engineering Materials \& Structures, 40 (4), 471-495. doi: http://doi.org/10.1111/ffe.12578

[8] Reiser, J., Wurster, S., Hoffmann, J., Bonk, S., Bonnekoh, C., Kiener, D. et. al. (2016). Ductilisation of tungsten (W) through cold-rolling: R-curve behaviour. International Journal of Refractory Metals and Hard Materials, 58, 22-33. doi: http://doi.org/ 10.1016/j.ijrmhm.2016.03.006

[9] Kumar, R. S. (2017). Crack-growth resistance behavior of mode-I delamination in ceramic matrix composites. Acta Materialia, 131, 511-522. doi: http://doi.org/10.1016/j.actamat.2017.04.012

[10] Griffith, A. (1920). The phenomena of rupture in solids. London: Philosophical Transactions of the Royal Society, $163-197$. doi: http://doi.org/10.1098/rsta.1921.0006

[11] Irwin, G.; Flugge, S. (Ed.) (1958). Fracture dynamics. Fracture of metals. Springer, 4, 551-590. doi: http://doi.org/10.1007/9783-662-43081-1_5 
[12] Babeshko, V. A., Evdokimova, O. V., Babeshko, O. M. (2019). A new type of cracks adding to griffith-irwin cracks. Doklady Physics, 64 (2), 102-105. doi: http://doi.org/10.1134/s1028335819030042

[13] Cherdantsev, N. V., Shadrin, A. V. (2017). A fluid pressure-loaded single crack located in a rock massif propagation trajectory calculation. Bulletin of Research Center for Safety in Coal Industry (Industial Safety), 4, 18-26. doi: http://doi.org/10.26631/ arc4-2017-18-26

[14] Mott, N. F. (2001). Electrons in disordered structures. Advances in Physics, 50 (7), 865-945. doi: http://doi.org/ $10.1080 / 00018730110102727$

[15] Ioffe, A. F. (1980). Problemy sovremennoi fiziki. Moscow: Nauka, 586.

[16] Sung, P.-H., Chen, T.-C. (2015). Studies of crack growth and propagation of single-crystal nickel by molecular dynamics. Computational Materials Science, 102, 151-158. doi: http://doi.org/10.1016/j.commatsci.2015.02.031

[17] Bleyer, J., Roux-Langlois, C., Molinari, J.-F. (2016). Dynamic crack propagation with a variational phase-field model: limiting speed, crack branching and velocity-toughening mechanisms. International Journal of Fracture, 204 (1), 79-100. doi: http:// doi.org/10.1007/s10704-016-0163-1

[18] Kobaiashi, T., Delli, D. (1981). Zavisimost mezhdu skorostiu treschiny i koeffitsientom intensivnosti napriazhenii v polimerakh s dvoinym lucheprelomleniem. Mekhanika razrusheniia. Moscow: Mir, 25, 101-119.

[19] Efimov, V. P., Sher, E. N. (2001). Opredelenie dinamicheskoi treschinostoikosti organicheskogo stekla. Prikladnaia mekhanika i tekhnicheskaia fizika, 42 (5), 217-225. Available at: https://www.sibran.ru/upload/iblock/947/9476bb624bblbc95b7dfe5316ea3b2c3.pdf

[20] Golubyatnikov, A. N. (2018). Small-amplitude discontinuities of solutions to equations of continuum mechanics. Proceedings of the Steklov Institute of Mathematics, 300 (1), 56-67. doi: http://doi.org/10.1134/s0371968518010041 